\title{
Vertical resolution dependence of gravity wave momentum flux simulated by an atmospheric general circulation model
}

\author{
S. Watanabe ${ }^{1}$, K. Sato ${ }^{2}$, Y. Kawatani ${ }^{1}$, and M. Takahashi ${ }^{3}$ \\ ${ }^{1}$ Japan Agency for Marine-Earth Science and Technology, Yokohama, Japan \\ ${ }^{2}$ Department of Earth and Planetary Science, Graduate School of Science, The University of Tokyo, Tokyo, Japan \\ ${ }^{3}$ Atmosphere and Ocean Research Institute, The University of Tokyo, Kashiwa, Japan \\ Correspondence to: S. Watanabe (wnabe@jamstec.go.jp)
}

Received: 19 September 2014 - Published in Geosci. Model Dev. Discuss.: 12 November 2014

Revised: 24 April 2015 - Accepted: 11 May 2015 - Published: 2 June 2015

\begin{abstract}
The dependence of the gravity wave spectra of energy and momentum flux on the horizontal resolution and time step of atmospheric general circulation models (AGCMs) has been thoroughly investigated in the past. In contrast, much less attention has been given to the dependence of these gravity wave parameters on models' vertical resolutions. The present study demonstrates the dependence of gravity wave momentum flux (GWMF) in the stratosphere and mesosphere on the model's vertical resolution, which is evaluated using an AGCM with a horizontal resolution of about $0.56^{\circ}$. We performed a series of sensitivity test simulations changing only the model's vertical resolution above a height of $8 \mathrm{~km}$, and found a global reduction of GWMF with increasing vertical resolution. Inertial gravity waves with short vertical wavelengths simulated at higher vertical resolutions might play an important role in determining GWMF in the summertime stratosphere. The sensitivity test simulation also demonstrated the importance of the model's vertical resolution on representing realistic behaviors of gravity waves near their critical level.
\end{abstract}

\section{Introduction}

Due to recent advancements in high-performance computing, simulations of the global atmosphere with sub-kilometer horizontal resolutions have been achieved, allowing convective systems to be resolved (Miyamoto et al., 2013). Considering the global momentum budget in such ultra-highresolution atmospheric models, explicitly resolved gravity waves (GWs) undoubtedly play important roles (Alexander et al., 2010).

The dependence of the GW spectra of energy and momentum flux on the horizontal resolution and time step of atmospheric general circulation models (AGCMs) has been studied in considerable depth. Using comprehensive atmospheric general circulation models, Koshyk and Hamilton (2001) and Hamilton et al. (2008) demonstrated the dependence of the horizontal wave-number spectra of GW energy on the horizontal resolution of the AGCM. Shutts and Vosper (2011) evaluated the dependence of GW energy on the horizontal resolution and time step of state-of-the-art numerical weather prediction models. These pioneering works have served as useful guidance to other modeling disciplines.

In contrast, much less attention has been given to the dependence of the GW parameters on the vertical resolution of the AGCM. At present, only a few AGCMs have achieved sub-kilometer vertical resolution coverage throughout the middle atmosphere, which is believed to be crucial for accurately reproducing GW-related processes such as generation, propagation, dissipation, and mixing. Among such high-vertical-resolution AGCMs, the model developed for the KANTO project had the highest vertical resolution of $300 \mathrm{~m}$, which was actually determined based on an expert judgment rather than a sensitivity test approach (Watanabe et al., 2008). The main purpose of the present study is to clarify the dependence of GW momentum flux on the vertical resolution of the model, with the aim of elucidating the optimum vertical resolution. 


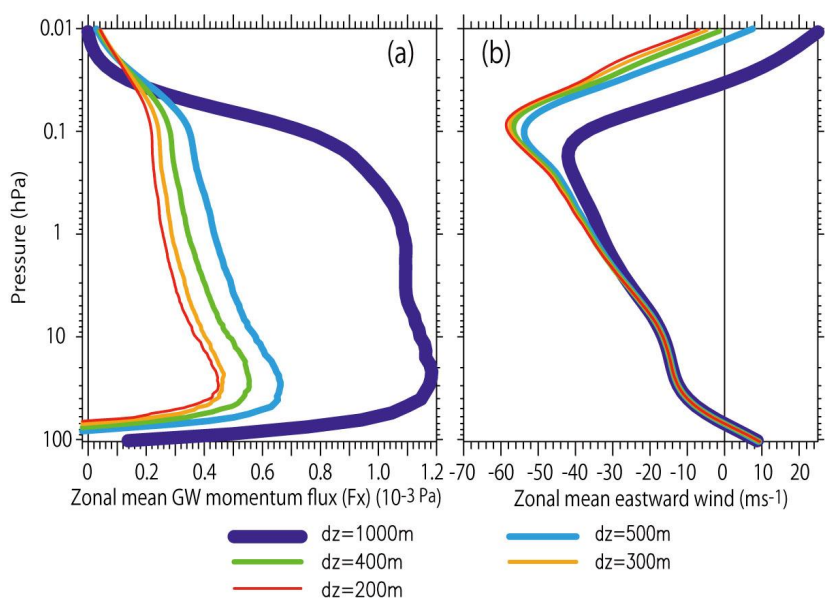

Figure 1. (a) Vertical profiles of the zonal mean net upward flux of the eastward momentum associated with the gravity wave component $\left(\lambda_{h}<950 \mathrm{~km}\right)$ for each vertical resolution. (b) Similar to (a) but for the zonal mean eastward winds. Averages over $35-40^{\circ} \mathrm{N}$ from 00:00 UT, 23 June, to 00:00 UT, 27 June are shown.

\section{Model and experimental design}

The model used in the present study was JAGUAR (Japanese Atmospheric General circulation model for Upper Atmosphere Research) (Watanabe and Miyahara, 2009). The vertical domain of this model extends from Earth's surface to a height of about $150 \mathrm{~km}$, although the present study focused on just the first $80 \mathrm{~km}$. The horizontal resolution was set to $0.56^{\circ}$, which is the same as that used by Watanabe et al. (2008) and Watanabe and Miyahara (2009). This horizontal resolution allows the model to resolve GWs with horizontal wavelengths larger than $\sim 190 \mathrm{~km}$. Considering the fact that the GWs observed in the mesosphere and lower thermosphere frequently include components with horizontal wavelengths in the order of $10 \mathrm{~km}$, the present model did not cover the full range of GW spectra. Note, however, that a series of studies has suggested that the model qualitatively reproduces the seasonal and interannual variations of large-scale thermal and wind structures, behaviors of explicitly resolved GWs and vertically fine structures of the extratropical tropopause layer (Watanabe et al., 2008; Watanabe and Miyahara, 2009; Tomikawa et al., 2008, 2012; Kawatani et al., 2010a, b; Sato et al., 2009, 2012; Miyazaki et al., 2010a, b).

In the present study, we performed a series of sensitivity test simulations changing only the model's vertical resolution above $8 \mathrm{~km}$. Vertical resolutions of $\mathrm{d} z=200,300,400$, 500, and $1000 \mathrm{~m}$ were used (Fig. A1). The vertical resolution below $8 \mathrm{~km}$ remained unchanged. (Changing the vertical resolution in the lower troposphere is known to have significant effects on the behaviors of physical parameterizations such as cumulus convection and boundary layer processes, but these are not addressed in the present study.) The vertical resolutions used in the KANTO project and the previ- ous JAGUAR study were 300 and $500 \mathrm{~m}$, respectively. The main focus in this study was GW momentum flux at northern summer mid-latitudes, where the short-term variability of planetary-scale and synoptic-scale waves is relatively small. This condition is important because it is preferable for GW source distribution (e.g., diabatic heating and jet-front systems) in the troposphere to be similar in each run, to a qualitative degree at the very least.

Due to the limited computational resources available for this study, we only performed a short-term deterministic forecast-type experiment starting from virtually the same initial condition as in the original (control) simulation. The original initial condition at 00:00 UT on 21 June was taken from a run using $\mathrm{d} z=500 \mathrm{~m}$, which had been spun-up for several years and well reproduced large-scale thermal and wind structures in the middle atmosphere. That initial condition was vertically interpolated into the $\mathrm{d} z=200,300,400$, and $1000 \mathrm{~m}$ runs, each of which was performed for the week of 21-27 June. The time step was set to $30 \mathrm{~s}$ in every run.

Meteorological fields, e.g., winds, temperatures, and precipitations, were output every $30 \mathrm{~min}$ as $30 \mathrm{~min}$ averages, and GW components were extracted using a high-pass filter based on the spherical harmonics with a cutoff horizontal wavelength of about $950 \mathrm{~km}$, i.e., wavelengths shorter than $950 \mathrm{~km}$ were extracted (Watanabe et al., 2008). GW momentum flux is referred to as GWMF hereafter for simplicity. This paper focuses on the net vertical flux of eastward momentum associated with the GW components, because it is of primary importance in the momentum budget in the middle atmosphere.

Both the chaotic behavior of the fluid system and the difference in the vertical resolution resulted in different evolutions of the synoptic motions and GW distributions. However, as will be shown later, the differences in the simulated GWMF in the middle atmosphere caused by the vertical resolution were significant. Since the simulated GW field requires only a few days for initial spin-up, i.e., to generate, propagate, and fill the stratosphere (Hamilton et al., 2008; Shutts and Vosper, 2011) the present work mainly focused on the period of 24-26 June, during which the synoptic systems in all five runs held a morphological resemblance to each other. Because the vertical interpolation used in the preparation of initial conditions disturbed the original dynamical state, spurious GWs appeared during the initial spin-up, though we found them to be negligible during the analysis period.

\section{Results}

\subsection{Strong dependence of GW momentum flux}

Figure 1a compares vertical profiles of the GWMF for northern summer mid-latitude, which were simulated using the five different vertical resolutions. Positive values suggest the 

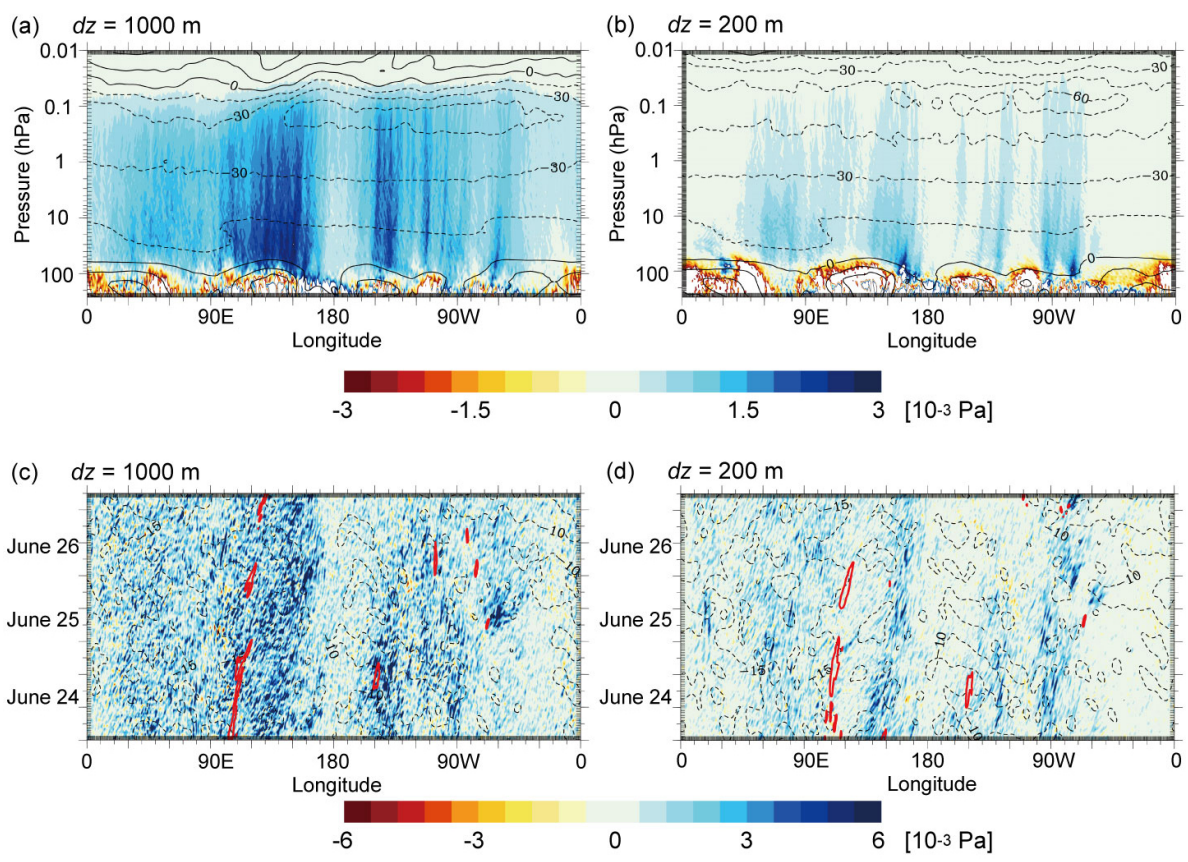

Figure 2. Upper panels: longitude-height distribution of net upward flux of the eastward momentum associated with the gravity wave component $\left(\lambda_{h}<950 \mathrm{~km}\right)$ in the $\mathrm{d} z=1000 \mathrm{~m}$ run (a) and $\mathrm{d} z=200 \mathrm{~m}$ run (b). The contours show low-pass filtered ( $\left.>1000 \mathrm{~km}\right)$ eastward winds with an interval of $15 \mathrm{~m} \mathrm{~s}^{-1}$. Averages over 35-40 $\mathrm{N}$ from 00:00 UT, 24 June, to 00:00 UT, 27 June, are shown. Lower panels: Hovmöller diagrams of the eastward gravity wave momentum flux (color) and the background large-scale eastward winds (black contours with an interval of $5 \mathrm{~m} \mathrm{~s}^{-1}$ ) at $30 \mathrm{hPa}$ in the $\mathrm{d} z=1000 \mathrm{~m}$ run (c) and $\mathrm{d} z=200 \mathrm{~m}$ run (d). Red contours show precipitation rate of $2 \mathrm{~mm} \mathrm{~h}{ }^{-1}$.

dominance of the GWs propagating upward and eastward against background easterly winds prevailing in the summertime stratosphere and mesosphere (e.g., Watanabe, 2008; Sato et al., 2009). It was quite surprising to find that the GWMF substantially decreases with increasing vertical resolution throughout the stratosphere and mesosphere. In other words, a coarse vertical resolution leads to a significant overestimation of the GWMF. This characteristic is opposite to that for horizontal resolution; a coarse horizontal resolution causes an underestimation of the GWMF, which results in a cold pole problem in the wintertime stratosphere (Hamilton et al., 1999). In these particular simulations, the dependence of the GWMF on the model's vertical resolution seems to start converging between $\mathrm{d} z=300 \mathrm{~m}$ and $\mathrm{d} z=200 \mathrm{~m}$. In the following sections, we discuss possible causes of this strong vertical resolution dependence.

\subsection{Critical level filtering}

Figure $1 \mathrm{~b}$ shows similar vertical profiles to Fig. 1a but for the zonal mean zonal wind. The wind profiles are almost identical to each other below $30 \mathrm{hPa}$, which implies that critical level filtering of GWs propagating upward due to background large-scale flows in the troposphere and lower stratosphere does not explain the differences in the GWMF. The difference in the zonal mean zonal wind above the $30 \mathrm{hPa}$ level rather reflects the difference in the GWMF, that is, the larger
GWMF in the coarser vertical resolution runs generally gives larger GW forcing in the upper stratosphere and mesosphere, decelerating large-scale winds through wave-mean flow interactions.

\subsection{Effects of particular GW events}

It is necessary to determine whether the present vertical resolution dependence of the GWMF originates from particularly severe GW events. The upper panels of Fig. 2 compare longitude-height distributions of the net eastward GWMF for $\mathrm{d} z=1000$ and $200 \mathrm{~m}$. It is revealed that the longitudinal distributions of large-scale zonal winds below the $30 \mathrm{hPa}$ level are qualitatively similar to each other. Meanwhile, the GWMF in the $\mathrm{d} z=1000 \mathrm{~m}$ run is generally larger than that in the $\mathrm{d} z=200 \mathrm{~m}$ run. The GWMF is large not only near active source regions $\left(100-160^{\circ} \mathrm{E}\right.$ and $\left.60-150^{\circ} \mathrm{W}\right)$ but also near relatively calm regions $\left( \pm 45^{\circ}\right.$ from $0^{\circ} \mathrm{E}$ and near the date line). Although differences in the tropospheric circulation and locations of convection in these two runs could account for the differences in the GWMF, we believe that the systematic and global differences in the GWMF cannot solely be explained by them. The lower panels of Fig. 2 show the time evolution of the GWMF at the $30 \mathrm{hPa}$ level, along with contours of strong precipitation. Strong GW events with large positive GWMF (e.g., $100-160^{\circ} \mathrm{E}$ ) are repeatedly observed in the east of strong precipitation regions (e.g., $100-120^{\circ} \mathrm{E}$ ), 

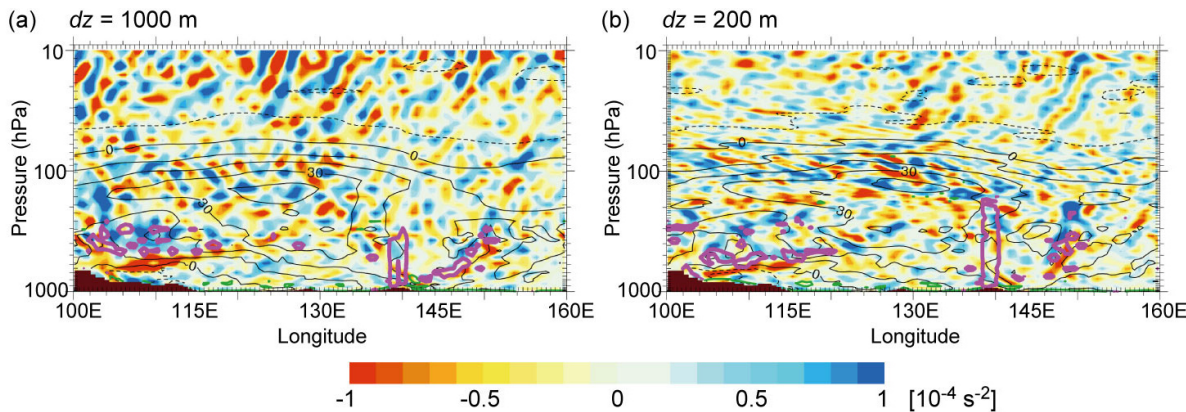

Figure 3. Instantaneous longitude-height distribution of the unfiltered divergence component of the horizontal winds (color), the low-pass filtered $\left(>1000 \mathrm{~km}\right.$ ) eastward winds (black contours with an interval of $15 \mathrm{~m} \mathrm{~s}^{-1}$ ) and the moist diabatic heating rate of $0.1 \mathrm{Kh}^{-1}$ (purple contours) at $37.5^{\circ} \mathrm{N}, 00: 00 \mathrm{UT}, 24 \mathrm{June}$. Green lines show regions of $R_{i}<0.25$, where parameterized turbulent mixing occurs in the model. Note that the longitudinal coverage is different from Fig. 2.
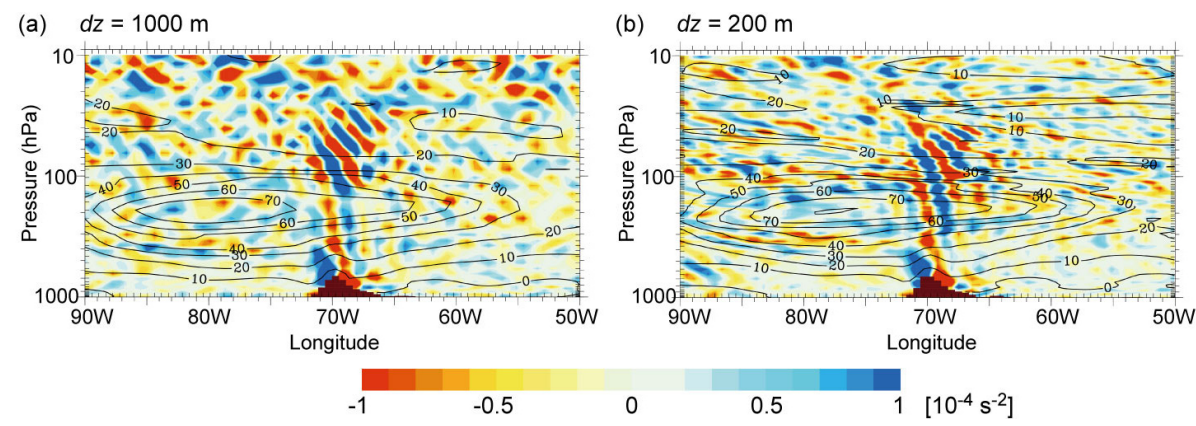

Figure 4. Instantaneous longitude-height distribution of the unfiltered divergence component of the horizontal winds (color), the low-pass filtered $\left(>1000 \mathrm{~km}\right.$ ) eastward winds (black contours with an interval of $10 \mathrm{~m} \mathrm{~s}^{-1}$ ) at $30^{\circ} \mathrm{S}, 06: 00 \mathrm{UT}, 27$ June.

suggesting that the GWMF is likely associated with convectively generated high-frequency GWs, which can penetrate westerly winds in the tropospheric sub-tropical jet (see the contours of the background zonal winds in the upper panels). Overall, it can be concluded that the vertical resolution dependence of the GWMF shown in Fig. 1 is not caused by any particular GW events but is likely caused by systematic differences related to GW behaviors.

\subsection{Effects of thin GWs}

Sato et al. (1999) reported the existence of a spectral peak of GWs near the inertial frequency in the global lower stratosphere of a high-resolution (about $1^{\circ}$ horizontal resolution) aqua-planet AGCM experiment, which was later confirmed by observational evidence (Sato and Yoshiki, 2008). Such GWs have the lowest intrinsic frequency among vertically propagating GWs and generally have short vertical wavelengths (e.g., less than $2-3 \mathrm{~km}$ ). Therefore, a coarse vertical resolution systematically reduces their number. Here, there is the apparent contradiction that the GWMF decreases in runs with higher vertical resolutions, in which thin GWs can be resolved.

Figure 3 shows close-up views of instantaneous longitudeheight GW distributions (horizontal wind divergence com- ponents). In the $\mathrm{d} z=200 \mathrm{~m}$ run, thin GWs $\left(\lambda_{z}=1-3 \mathrm{~km}\right)$ which are likely emitted by convective heating at around $140^{\circ} \mathrm{E}$ propagate upward and westward against the tropospheric westerly jet. Qualitatively, these low-frequency GWs generated within the sub-tropical jet reach their own critical levels near the zero-wind contours below a $50 \mathrm{hPa}$ level, and do not directly affect the GWMF observed above the $30 \mathrm{hPa}$ level.

As expected, the thin GWs $\left(\lambda_{z}=1-3 \mathrm{~km}\right)$ in the lower stratosphere are absent in the $\mathrm{d} z=1000 \mathrm{~m}$ run. Instead, GWs with $\lambda_{z}>4-5 \mathrm{~km}$ are dominant everywhere, and the amplitude of the GWs seen above the $30 \mathrm{hPa}$ level is obviously larger than those in the $\mathrm{d} z=200 \mathrm{~m}$ run. Although the tropospheric circulation, distribution of moist diabatic heating, and phase structures of GWs in the lower troposphere are not quite similar in the two runs, these circumstances may imply the existence of suppression effects of the thin GWs in the $\mathrm{d} z=200 \mathrm{~m}$ run, which effectively suppress the high-frequency GWs with longer vertical wavelengths. One possible candidate of the suppression mechanisms is the parameterized turbulent diffusion, which is not obviously seen in Fig. 3 but sometime induced by wave saturation of thin GWs. The GWs with longer vertical wavelength encountering the turbulent layer would be suppressed due to 


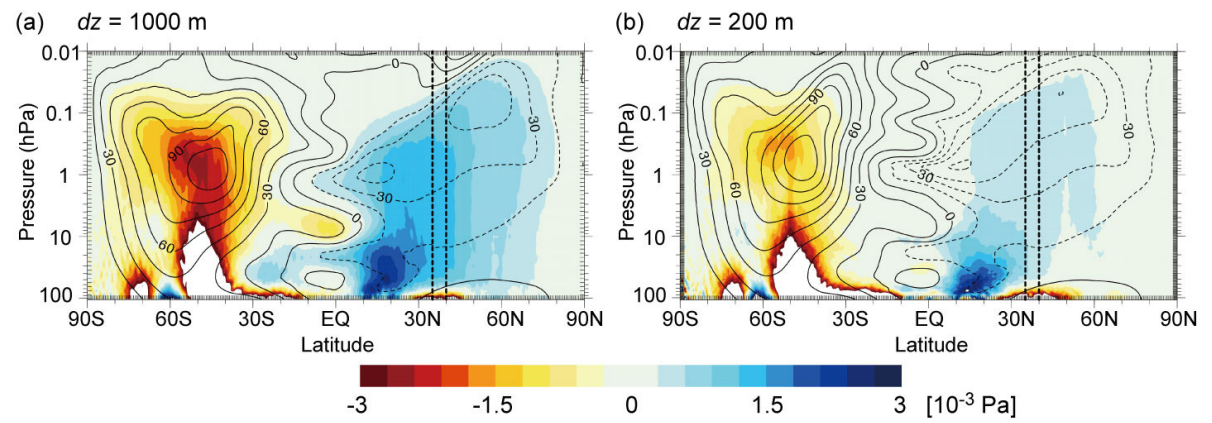

Figure 5. The zonal mean eastward winds (contours) and net upward flux of the eastward momentum associated with the gravity wave component $\left(\lambda_{h}<950 \mathrm{~km}\right)$ in the $\mathrm{d} z=1000 \mathrm{~m}$ (a) and $\mathrm{d} z=200 \mathrm{~m}$ (b) simulations. Averages from 00:00 UT, $23 \mathrm{June}$, to 00:00 UT, $27 \mathrm{June}$, are shown. The color shading with momentum flux larger than $\pm 3 \times 10^{-3} \mathrm{~Pa}$ are omitted. The latitude box of $35-40^{\circ} \mathrm{N}$ is indicated, which we mainly focus on in the present study.

vertical redistribution of wave momentum. Another suppression mechanism might be a discontinuous vertical profile of the buoyancy frequency, which causes the partial reflection of GWs (e.g., Sato et al., 2012).

More statistical investigations are necessary to clarify the roles of thin GWs in the high-vertical-resolution models. One relevant study would be Lane and Knievel (2005), who investigated the vertical resolution dependence of convectively generated GWs by using a mesoscale model with much finer horizontal resolutions. Their study revealed that the behavior of gravity waves as resolution is increased is not a straightforward monotonic behavior.

\subsection{Orographic GWs in the winter hemisphere}

We have mainly focused on the non-orographic GWs appearing in the northern summer mid-latitudes. Here we briefly investigate a case for orographic GWs in the southern winter mid-latitudes. Figure 4 shows a similar longitude-height GW distribution to Fig. 3 but over the Andes. Westerly winds over the surface orography excite deep GWs, and their phase structures resemble each other below about $100 \mathrm{hPa}$. Above $100 \mathrm{hPa}$ of the $\mathrm{d} z=200 \mathrm{~m}$ run, the vertical wavelength of orographic GWs substantially decreases with height and the GWs disappear above about $30 \mathrm{hPa}$. This behavior suggests occurrences of Doppler shifting and dissipation near the critical level associated with vertical variations of the background winds. A weak wind layer is observed at $20-50 \mathrm{hPa}$ downwind of the mountain, implying effects of wave-mean flow interactions associated with the orographic GWs. On the other hand, the minimum vertical wavelength of orographic GWs in the $\mathrm{d} z=1000 \mathrm{~m}$ run is $2 \mathrm{~km}$, and they propagate higher than those seen in the $\mathrm{d} z=200 \mathrm{~m}$ run. The temporary and regional average of GWMF during this particular orographic $\mathrm{GW}$ event in the $\mathrm{d} z=200 \mathrm{~m}$ run was smaller than that in the $\mathrm{d} z=1000 \mathrm{~m}$ run throughout the troposphere and stratosphere (not shown). Although such dependence is similar to the northern summer mid-latitudes, longer simu- lations and careful examinations are needed since mountain waves have large intermittency, having just one case during the present 1 -week simulation.

\subsection{Latitudinal variations}

Figure 5 compares meridional distributions of the zonal mean zonal winds and GWMF, which were simulated in the $\mathrm{d} z=$ $1000 \mathrm{~m}$ and $\mathrm{d} z=200 \mathrm{~m}$ simulations. At almost all latitudes, the magnitude of GWMF decreases with increasing vertical resolution, which in turn alters the zonal mean wind structures through changes in $\mathrm{GW}$ forcing. Longer simulations are required to obtain climatological views of the GWMF distributions and background wind structures, and their dependence on the model's vertical resolution.

\section{Concluding remarks}

In order to investigate the vertical resolution dependence of the GWMF in a comprehensive AGCM which explicitly resolves a portion of the GW spectra $\left(\lambda_{h}>190 \mathrm{~km}\right)$, a series of sensitivity test simulations was performed changing only the model's vertical resolution $(\mathrm{d} z=200,300,400,500$, and $1000 \mathrm{~m}$ ). The simulated GWMF in the stratosphere and mesosphere of northern summertime mid-latitudes showed a strong dependence on the model's vertical resolution. The GWMF was systematically overestimated with decreasing vertical resolution; it was confirmed that this overestimation did not stem from any particular GW events. The existence of low-frequency thin $\left(\lambda_{z}=1-3 \mathrm{~km}\right) \mathrm{GWs}$ in the lower stratosphere might cause the vertical resolution dependence of the GWMF. On the other hand, the case study for the deep orographic GWs in the southern wintertime mid-latitude showed the importance of vertical resolution on appropriate representation of wave-phase tilting and dissipation near the critical level. The comparison for the meridional distribution of GWMF simulated with different vertical resolutions confirmed that the GWMF in the middle atmosphere decreased 
with increasing vertical resolution at all latitudes. Further discussions and investigations are required to obtain plausible mechanisms that explain the causality of the present findings. 
Appendix A

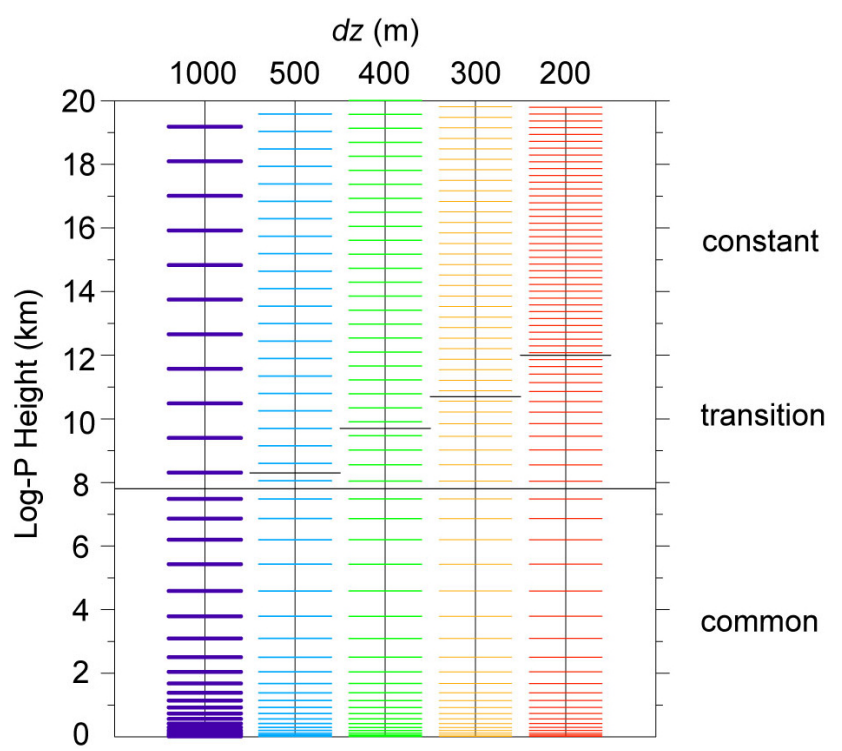

Figure A1. Standard vertical layer distribution for each vertical resolution. Note that a hybrid sigma-pressure coordinate system was actually used in the model, and a scale height of $7 \mathrm{~km}$ was used to calculate the log-pressure standard levels shown in this figure. 
Author contributions. S. Watanabe developed the model code. S. Watanabe and K. Sato designed the experiments, and S. Watanabe carried them out. S. Watanabe prepared the manuscript with contributions from all co-authors.

Acknowledgements. The authors would like to thank two anonymous referees for providing helpful comments on the original manuscript. This study was partly supported by a Grant-in-Aid for Scientific Research (A) 25247075 and the SOUSEI Program, MEXT, Japan. The numerical simulations in this study were performed using the Earth Simulator, and figures were drawn using GTOOL and the GFD-DENNOU Library.

Edited by: O. Marti

\section{References}

Alexander, M. J., Geller, M., McLandress, C., Polavarapu, S., Preusse, P., Sassi, F., Sato, K., Eckermann, S., Ern, M., Hertzog, A., Kawatani, Y., Pulido, M., Shaw, T. A., Sigmond, M., Vincent, R., and Watanabe, S.: Recent developments in gravitywave effects in climate models and the global distribution of gravity-wave momentum flux from observations and models, Q. J. Roy. Meteor. Soc., 136, 1103-1124, doi:10.1002/qj.637, 2010.

Hamilton, K., Wilson, R. J., and Hemler, R. S.: Middle atmosphere simulated with high vertical and horizontal resolution versions of a GCM: improvements in the cold pole bias and generation of a QBO-like oscillation in the tropics, J. Atmos. Sci., 56, 38293846, 1999.

Hamilton, K., Takahashi, Y. O., and Ohfuchi, W.: Mesoscale spectrum of atmospheric motions investigated in a very fine resolution global general circulation model, J. Geophys. Res., 113, D18110, doi:10.1029/2008jd009785, 2008.

Kawatani, Y., Sato, K., Dunkerton, T. J., Watanabe, S., Miyahara, S., and Takahashi, M.: The Roles of Equatorial Trapped Waves and Internal Inertia-Gravity Waves in Driving the Quasi-Biennial Oscillation. Part I: Zonal Mean Wave Forcing, J. Atmos. Sci., 67, 963-980, doi:10.1175/2009JAS3222.1, 2010a.

Kawatani, Y., Sato, K., Dunkerton, T. J., Watanabe, S., Miyahara, S., and Takahashi, M.: The Roles of Equatorial Trapped Waves and Internal Inertia-Gravity Waves in Driving the Quasi-Biennial Oscillation. Part II: Three-Dimensional Distribution of Wave Forcing, J. Atmos. Sci., 67, 981-997, doi:10.1175/2009JAS3223.1, $2010 b$.

Koshyk, J. N. and Hamilton, K.: The horizontal kinetic energy spectrum and spectral budget simulated by a high-resolution troposphere-stratosphere-mesosphere GCM, J. Atmos. Sci., 58, 329-348, 2001.

Lane, T. P. and Knievel, J. C.: Some effects of model resolution on simulated gravity waves generated by deep, mesoscale convection, J. Atmos. Sci., 62, 3408-3419, 2005.

Miyamoto, Y., Kajikawa, Y., Yoshida, R., Yamaura, T., Yashiro, H., and Tomita, H.: Deep moist atmospheric convection in a subkilometer global simulation, Geophys. Res. Lett., 40, 4922-2926, doi:10.1002/grl.50944, 2013.

Miyazaki, K., Watanabe, S., Kawatani, Y., Sato, K., Tomikawa, Y., and Takahashi, M.: Transport and Mixing in the Extratropical Tropopause Region in a High-Vertical-Resolution GCM. Part
II: Relative Importance of Large-Scale and Small-Scale Dynamics, J. Atmos. Sci., 67, 1315-1336, doi:10.1175/2009JAS3334.1, 2010a.

Miyazaki, K., Watanabe, S., Kawatani, Y., Tomikawa, Y., Takahashi, M., and Sato, K.: Transport and Mixing in the Extratropical Tropopause Region in a High-Vertical-Resolution GCM. Part I: Potential Vorticity and Heat Budget Analysis, J. Atmos. Sci., 67, 1293-1314, doi:10.1175/2009JAS3221.1, 2010b.

Sato, K., Kumakura, T., and Takahashi, M.: Gravity waves appearing in a high-resolution GCM simulation, J. Atmos. Sci., 56, 1005-1018, doi:10.1175/15200469(1999)056<1005:GWAIAH>2.0.CO;2, 1999.

Sato, K., Watanabe, S., Kawatani, Y., Tomikawa, Y., Miyazaki, K., and Takahashi, M.: On the origins of mesospheric gravity waves, Geophys. Res. Lett., 36, L19801, doi:10.1029/2009GL039908, 2009.

Sato, K., Tateno, S., Watanabe, S., and Kawatani, Y.: Gravity Wave Characteristics in the Southern Hemisphere Revealed by a High-Resolution Middle-Atmosphere General Circulation Model, J. Atmos. Sci., 69, 1378-1396, doi:10.1175/JAS-D-110101.1, 2012.

Satoh, M., Tomita, H., Yashiro, H., Miura, H., Kodama, C., Seiki, T., Noda, A., Yamada, Y., Goto, D., Sawada, M., Miyoshi, T., Niwa, Y., Hara, M., Ohno, T., Iga, S.-I., Arakawa, T., Inoue, T., and Kubokawa, H.: The Non-hydrostatic Icosahedral Atmospheric Model: description and development, Progress in Earth and Planetary Science, 1, 18, doi:10.1186/s40645-014-0018-1, 2014.

Shutts, G. J. and Vosper, S. B.: Stratospheric gravity waves revealed in NWP model forecasts, Q. J. Roy. Meteor. Soc., 137, 303-317, doi:10.1002/qj.763, 2011.

Tomikawa, Y., Sato, K., Watanabe, S., Kawatani, Y., Miyazaki, K., and Takahashi, M.: Wintertime temperature maximum at the subtropical stratopause in a T213L256 GCM, J. Geophys. Res., 113, D17117, doi:10.1029/2008JD009786, 2008.

Tomikawa, Y., Sato, K., Watanabe, S., Kawatani, Y., Miyazaki, K., and Takahashi, M.: Growth of planetary waves and the formation of an elevated stratopause after a major stratospheric sudden warming in a T213L256 GCM, J. Geophys. Res., 117, D16101, doi:10.1029/2011JD017243, 2012.

Watanabe, S.: Constraints on a non-orographic gravity wave drag parameterization using a gravity wave resolving general circulation model, Sci. Online Lett. Atmos., 4, 61-64, doi:10.2151/sola.2008-016, 2008.

Watanabe, S. and Miyahara, S.: Quantification of the gravity wave forcing of the migrating diurnal tide in a gravity wave-resolving general circulation model, J. Geophys. Res., 114, D07110, doi:10.1029/2008JD011218, 2009.

Watanabe, S., Kawatani, Y., Tomikawa, Y., Miyazaki, K., Takahashi, M., and Sato, K.: General aspects of a T213L256 middle atmosphere general circulation model, J. Geophys. Res., 113, D12110 doi:10.1029/2008jd010026, 2008.

Watanabe, S., Tomikawa, Y., Sato, K., Kawatani, Y., Miyazaki, K., and Takahashi, M.: Simulation of the eastward 4-day wave in the Antarctic winter mesosphere using a gravity wave resolving general circulation model, 114, D16111, doi:10.1029/2008JD011636, 2009. 